\title{
Resonance absorption, reflection, transmission of phonons and heat transfer through interface between two solids
}

\author{
Yu.A. Kosevich \\ Semenov Institute of Chemical Physics, Russian Academy of Sciences, 4 Kosygina Str., Moscow 119991, Russia \\ E-mail: kosevich@polymer.chph.ras.ru
}

\author{
A. Feher \\ Centre of Low Temperature Physics Faculty of Science P.J. Sáfárik University \& Institute of Experimental Physics \\ SAS, Park Angelinum 9, Kośice 041 54, Slovakia \\ E-mail: alexander.feher@upjs.sk \\ E.S. Syrkin \\ B. Verkin Institute for Low Temperature Physics and Engineering of the National Academy of Sciences of Ukraine \\ 47 Lenin Ave., Kharkov 61103, Ukraine \\ E-mail: syrkin@ilt.kharkov.ua \\ Received February 14, 2008
}

\begin{abstract}
The different mechanisms of resonant transport of phonons between two media in the presence of impurity intermediate layer are described. Particular attention is focused on the resonance interaction of elastic waves with a two-dimensional defect on the contact boundary between two solids, on the multichannel interface phonon scattering and on the experimentally observed nonmonotonic temperature dependence of the reduced heat flux. In the cases when there is a direct interaction between edge atoms of the matrix as non-nearest neighbors or when the impurities do not fill completely the 2D interface layer, the additional channel for the transmission of phonons through the interface opens. This additional transmission channel manifests itself as a transmission (or reflection or absorption) peak with an asymmetric line shape (the so-called Fano resonance for phonons due to interference between the two transmission channels). Some applications of the Fano effect in magnon heat conductivity are also discussed.
\end{abstract}

PACS: 63.20.-e Phonons in crystal lattices.

Keywords: interface, quasi-local frequency, ballistic transport, Fano resonance.

\section{Introduction}

Arnold Markovich Kosevich has devoted much attention to the investigation of the physical phenomena connected with the presence of weakly bounded local and extended (two-dimensional planar) defects in a crystal lattice. First articles in this field were published by A.M. Kosevich together with his scientific teacher I.M. Lifshits [1], and with his pupil V.I. Khokhlov [2] in the 60th of the last century. Our work is devoted to the investigation of resonance mechanisms of transmission, reflection and absorption of phonons and phonon heat transfer through the interface between two crystal lattices, taking into account the dynamics of the intermediate impurity layer.

The presence of the planar defect has a substantial influence on various dynamic, thermodynamic and kinetic characteristics of crystals. To study this influence, it is necessary to elucidate the features of the interaction of phonons with the planar defect. The studies of resonance effects in the scattering of acoustic waves from and formation of localized and resonance vibrational states on a planar defect have a considerable interest [3-9], since such effects can give rise to the features in the kinetic 
phonon characteristics of the intercrystalline interfaces which can be experimentally observed.

\section{Interaction of acoustic waves with interface}

A.M. Kosevich has proposed to consider basic properties of the resonance transmission with the example of one-dimensional model [9]. One-dimensional (1D) models, despite their simplicity, can give qualitative description of many physical phenomena observed in real three-dimensional (3D) systems. Besides that, in the case of normal incidence of acoustic wave on the interface, the results received for 1D model coincide with the results received for 3D model up to a numerical factor.

Following the model proposed in Ref. 5, we consider 1D oscillator chain with a point defect with complex structure of local inter-atomic force constants as a one-dimensional projection of the two-dimensional (planar) defect, see Fig. 1. Such one-dimensional model can be applied to the normal incidence at the planar defect in three-dimensional crystal of the acoustic wave, which propagates along the symmetry axis of the crystal. In this case we can assume that there is no change of the acoustic wave polarization at the interface. We introduce the following notations: $m_{1}$ and $\gamma_{1}$ are the mass of an atom and the force constant in the host linear chain, $m_{2}$ and $\gamma_{2}$ are the mass of an impurity atom and the force constant characterizing its coupling with the host chain, $\gamma_{3}$ is the force constant which describes the direct interaction between (non-nearest neighboring) edge atoms of the matrix, passing over the defect atom [5], see Fig. 1.

Let a wave incident on a defect be described by the expression: $u(n)=u(0) \exp (i k n)$, where $n$ enumerates the atoms in the chain, $n=0$ corresponds to the defect atom. The reflected wave can be written as $u_{r}(n)=r u(0) \exp (-i k n)$, transmitted wave can be written through amplitude coefficient $t$, correspondingly. Here $r$ and $t$ are, respectively, the complex reflection and transmission amplitudes of the incident acoustic wave. First we consider the case of the defect with $\gamma_{3}=0$ (without an additional phonon pass). Using equations of motion of a linear chain containing such a defect, one can obtain the following expressions for $r$ and $t$ :

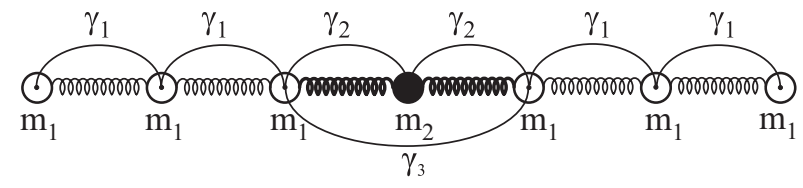

Fig. 1. Simple 1D model of a planar lattice defect with locally different nearest-neighbor and non-nearest neighbor interactions (with force constants $\gamma_{2}$ and $\gamma_{3}$ ) in the vicinity of the defect.

$$
r=\frac{\left[\left(\gamma_{2}-\gamma_{1}\right) m_{1} \varepsilon-\gamma_{2}^{2}\right]\left(m_{2} \varepsilon-2 \gamma_{2}\right)+\gamma_{2}^{2}\left(m_{1} \varepsilon-2 \gamma_{2}\right)}{\left\{\gamma_{1}[q(\varepsilon)-1]\left(m_{2} \varepsilon-2 \gamma_{2}\right)-m_{2} \gamma_{2} \varepsilon q(\varepsilon)\right\}\left\{\gamma_{1}[q(\varepsilon)]\right\}},
$$

$$
t=\frac{\gamma_{2}^{2} \sqrt{\varepsilon m_{1}\left(4 \gamma_{1}-\varepsilon m_{1}\right)}}{\left\{\gamma_{1}[q(\varepsilon)-1]\left(m_{2} \varepsilon-2 \gamma_{2}\right)-m_{2} \gamma_{2} \varepsilon q(\varepsilon)\right\}\left\{\gamma_{1}[q(\varepsilon)]\right\}},
$$

where $\varepsilon \equiv \omega^{2}$ is the square of the vibrational frequency of atoms in the chain and $q(\varepsilon)$ is the function defined by the expression:

$$
q(\varepsilon)=1-\frac{\varepsilon m_{1}}{2 \gamma_{1}}+i\left(\frac{\varepsilon m_{1}}{\gamma_{1}}\right)^{1 / 2}\left(1-\frac{\varepsilon m_{1}}{4 \gamma_{1}}\right)^{1 / 2} .
$$

We are interested in the features of the numerator in Eq. (1). Vanishing of the numerator corresponds to the resonant transmission of the wave through the impurity (impurity monolayer). The squared frequency satisfying this condition is

$$
\varepsilon_{\mathrm{res}}=\frac{2 \gamma_{1} \gamma_{2} m_{1}-\gamma_{2}^{2}\left(m_{1}+m_{2}\right)}{\left(\gamma_{1}-\gamma_{2}\right) m_{1} m_{2}} .
$$

For many systems, particularly for point contacts, the coupling constant of the defect to the host lattice is typically much smaller than the coupling constant in the host chain its $\gamma_{2} / \gamma_{1}<<1$. In this case the following expression for $\varepsilon_{\text {res }}$ is valid:

$$
\varepsilon_{\text {res }}=2 \frac{\gamma_{2}}{m_{2}}+\frac{\gamma_{2}}{\gamma_{1}}\left(\frac{1}{m_{2}}-\frac{1}{m_{1}}\right) \approx 2 \frac{\gamma_{2}}{m_{2}} .
$$

The resonant wave transmission arises owing to the interaction of the incident wave with an eigenmode of the chain formed due to the presence of the impurity. Up to the small terms of the order of $\gamma_{2} / \gamma_{1}$, the frequency of this vibrational mode is determined solely by the interaction of the defect with the host lattice and by the mass of this defect. Within this model we obtain that outside of very narrow frequency region, the wave incident on the defect is almost completely reflected, i.e., the reflection coefficient is close to unity, and the transmission coefficient is very small, see Fig. 2. At the resonance frequency, the reflection coefficient goes to zero and the transmission coefficient goes to unity.

The resonance frequency describes a low frequency, weak-dispersion mode of the optical type. The amplitude of the displacements of the impurity atom in this mode is significantly greater than the amplitude of the vibrations of the atoms of the host lattice. We note that the corresponding results in the long-wave limit were received in Refs. 3 and 4 with the use of the theory of capillary phenomena in the theory of elasticity, the main features of which was developed in Refs. 5, 10. 


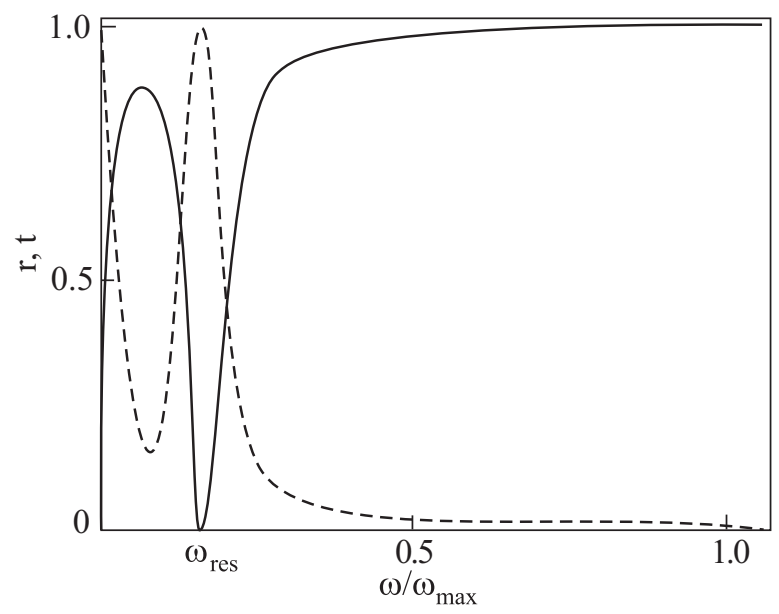

Fig. 2. Amplitude coefficients of wave reflection $r$ (solid curve) and transmission $t$ (dashed curve) versus the frequency of the incident wave for crystals with identical physical properties; $\omega_{\max }$ is the maximum frequency of continuous spectrum of the system.

\section{Phonon heat transport through thin film}

In the previous section a mechanism of the resonance phonon transmission through the planar defect was described under the assumption of a single resonance (quasi-local) frequency. This means that the intermediate layer is homogeneous along all the area of the contact both in the impurity contents and in the thickness. As for the real experiments, it is necessary to take into account that, as a rule, the contact surface is a set of sections in which the contents of impurities and thicknesses of the layers could be different. It is possible to assume in this case that the different channels of the phonon transport correspond to different sections of the flat layer. Every channel possesses its own resonance frequency $\omega_{\text {res }}=\sqrt{\varepsilon_{\text {res }}}$. When the phonon transport is considered at temperatures above $2 \mathrm{~K}$, this transport obeys rules of the geometric optics [8]. Therefore, the total phonon heat flow is the sum of the flows in every channel. Let $a_{n}$ be the area of a part of the corresponding contact with number $n$ and $D_{n}(\omega)$ the phonon energy transmission coefficient for the $n$th channel, characterized by the resonance frequency of transmission $\omega_{n}$. Then the effective coefficient of multichannel resonance transmission of phonons is described by the expression [8]

$$
D(\omega)=\frac{\sum_{n}^{n_{\max }} D_{n}(\omega) a_{n}}{\sum_{n=1}^{n_{\max }} a_{n}} .
$$

The capillary theory [5] of phonon transport makes it possible to take account of different types of impurities and different channel thicknesses using a single parameter $\rho_{s}$. Assuming all channels to be uniform with respect to the impurity composition, we can write $\rho_{s n}=\rho_{s 1} n$, where $\rho_{s 1}$ is the excess surface density of the molecular layer with smallest thickness and $n$ is the number of molecular layers in the channel, which serves as the channel number.

Consider two anisotropic body centered (BC) lattices with an intercalated interface layer. We choose that the axes $\mathrm{O} X$ and $\mathrm{O} Y$ are parallel to the boundary. Let's $a$ is the distance between neighbor atoms at the plane $X \mathrm{O} Y$, and $b / 2$ is the distance between neighbor atoms layers perpendicular to the axis $\mathrm{OZ}$ (see Fig. 3). The system is homogeneous in the plane $X \mathrm{O} Y$. Using the standard representation for the displacement $u(t, x, y, z)=u(z) \exp \left(-i \omega t+i k_{x} x / a+i k_{y} y / a\right)$ we have the equations of the movement of atoms:

$$
\begin{gathered}
m(z) \varepsilon u(z)+2 \alpha(z)\left[\cos k_{x}+\cos k_{y}-2\right] u(z)+ \\
+4 \gamma(z, z+b / 2)\left[\cos \left(k_{x} / 2\right) \cos \left(k_{y} / 2\right) u(z+b / 2)-u(z)\right]+ \\
+4 \gamma(z, z-b / 2)\left[\cos \left(k_{x} / 2\right) \cos \left(k_{y} / 2\right) u(z-b / 2)-u(z)\right]=0,
\end{gathered}
$$

where $\varepsilon=\omega^{2}, \omega$ is the vibration frequency, $m(z)$ is an atom mass, $\alpha(z)$ is the intra-layer interaction, and $\gamma(z, z+b / 2)$ is the interlayer interaction between neighboring layers. In our case

$$
\begin{gathered}
m(z)=m_{1} \text { for } z \leq-d / 2, \\
m(z)=m_{2} \text { for }-d / 2<z<d / 2,
\end{gathered}
$$

$m(z)=m_{3}$ for $d / 2 \leq z ; \alpha(z)=\alpha_{1}$ for $z \leq-d / 2$,

$$
\begin{gathered}
\alpha(z)=\alpha_{2} \text { for }-d / 2<z<d / 2, \alpha(z)=\alpha_{3} \text { for } d / 2 \leq z ; \\
\gamma(z, z+b / 2)=\gamma_{1} \text { for } z \leq-(d+b) / 2 \\
\gamma(z, z+b / 2)=\gamma_{2} \text { for }-(d+b) / 2<z<d / 2 \\
\gamma\left(z, z+b / 2=\gamma_{3} \text { for } d / 2 \leq z .\right.
\end{gathered}
$$

There are following equations for each lattice:

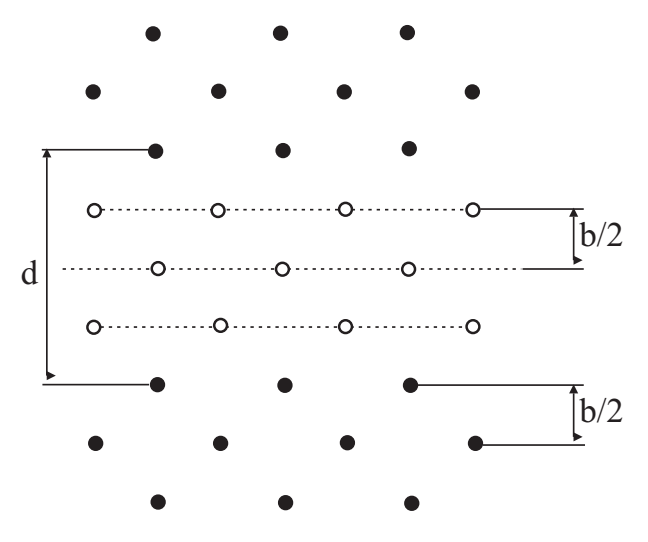

Fig. 3. Schematic figure showing an interface between two crystal lattices which contain three intercalant impurity layers. 


$$
\begin{gathered}
m_{n} \varepsilon u_{n}(z)+2 \alpha_{n}\left[\cos k_{x}+\cos k_{y}-2\right] u_{n}(z)+ \\
+4 \gamma_{n}\left[\cos \left(k_{x} / 2\right) \cos \left(k_{y} / 2\right) u(z+b / 2)-u(z)\right]+ \\
+4 \gamma_{n}\left[\cos \left(k_{x} / 2\right) \cos \left(k_{y} / 2\right) u(z-b / 2)-u(z)\right]=0, \\
n=1,2,3 .
\end{gathered}
$$

We assume bulk excitations in the first and the third lattices

$z \leq-d / 2: u(z)=A_{1} \exp \left(2 i k_{1 z} z / b\right)+B_{1} \exp \left(-2 i k_{1 z} z / b\right)$,

$-d / 2 \leq z \leq d / 2: u(z)=A_{2} \exp \left(2 k_{2 z} z / b\right)+B_{2} \exp \left(-2 k_{2 z} z / b\right)$,

$z \geq d / 2: u(z)=A_{3} \exp \left(2 i k_{3 z} z / b\right)$,

where $A_{1}, B_{1}$ and $A_{3}$ are the amplitudes of initial, reflected and transmitted waves.

The frequency dependences of transmission coefficients $D=A_{3} / A_{1}$ for different thickness $d$ in the considered model are represented in Fig. 4.

The above theory can be developed for the multichannel resonance transport of phonons across the interface between two media and can be applied for the interpretation of experimental measurements of the phonon ballistic transport in the point contacts $\mathrm{Si}-\mathrm{Cu}[11,12]$. These works have revealed for the first time the low-temperature quantum ballistic transport of phonons in the temperature region from 0.1 to $3 \mathrm{~K}$. Besides that, in papers $[11,12]$ a reduced point contact heat flow in the regime of the geometric optics for phonons was measured in the temperature interval from 3 to $10 \mathrm{~K}$. The results obtained in these experiments have shown that in this temperature interval the reduced heat flow through the point contact is a nonmonotonous function of temperature and has pronounced peaks at temperatures $T_{1}=4.46 \mathrm{~K}, T_{2}=6.53 \mathrm{~K}$, $T_{3}=8.77 \mathrm{~K}$. We suppose that the series of the peaks of the reduced heat flow could be explained $[11,12]$ by the model presented in Fig. 5.

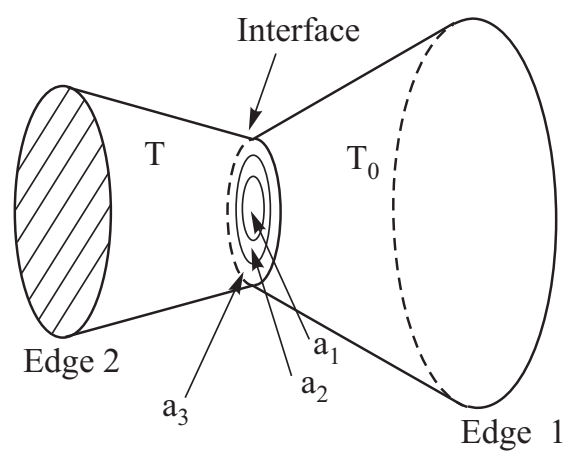

Fig. 5. Schematic model of a point contact. $T$ and $T_{0}$ are temperatures of the massive edges of the contact, $a_{1}, a_{2}$, and $a_{3}$ are zones with different composition of the interface layer.

Observed peaks are the result of the resonance phonon transport. In the case of the single-channel resonance transport studied in Ref. 13, a model of the narrow resonance peak was applied, meaning the following: the total heat flux $\dot{Q}$ may be then written as the sum of the ballistic flux $\dot{Q}_{B}$ and the resonance heat flux $\dot{Q}_{R}, \dot{Q}=\dot{Q}_{B}+\dot{Q}_{R}$. Assuming the narrow resonance peak near the frequency $\omega_{0}^{\alpha}$, we obtain the following formula describing the temperature dependence of the heat flux:

$$
\begin{gathered}
\dot{Q}\left(T, T_{0}\right)=C\left(T^{4}-T_{0}^{4}\right)+ \\
+\sum_{\alpha} K_{\alpha}\left[\frac{1}{\exp \left(\hbar \omega_{0}^{\alpha} / T\right)-1}-\frac{1}{\exp \left(\hbar \omega_{0}^{\alpha} / T_{0}\right)-1}\right]
\end{gathered}
$$

To separate the two parts of the total heat flux, its value can be divided by $\left(T^{4}-T_{0}^{4}\right)$. This model (using only one frequency) can be fitted to our experimental data with correlation of about 0.95 . The resonance frequency $\omega_{0}$ is connected with $T_{\max }$ by the relation $h \omega_{0}=3.89 T_{\max }$.
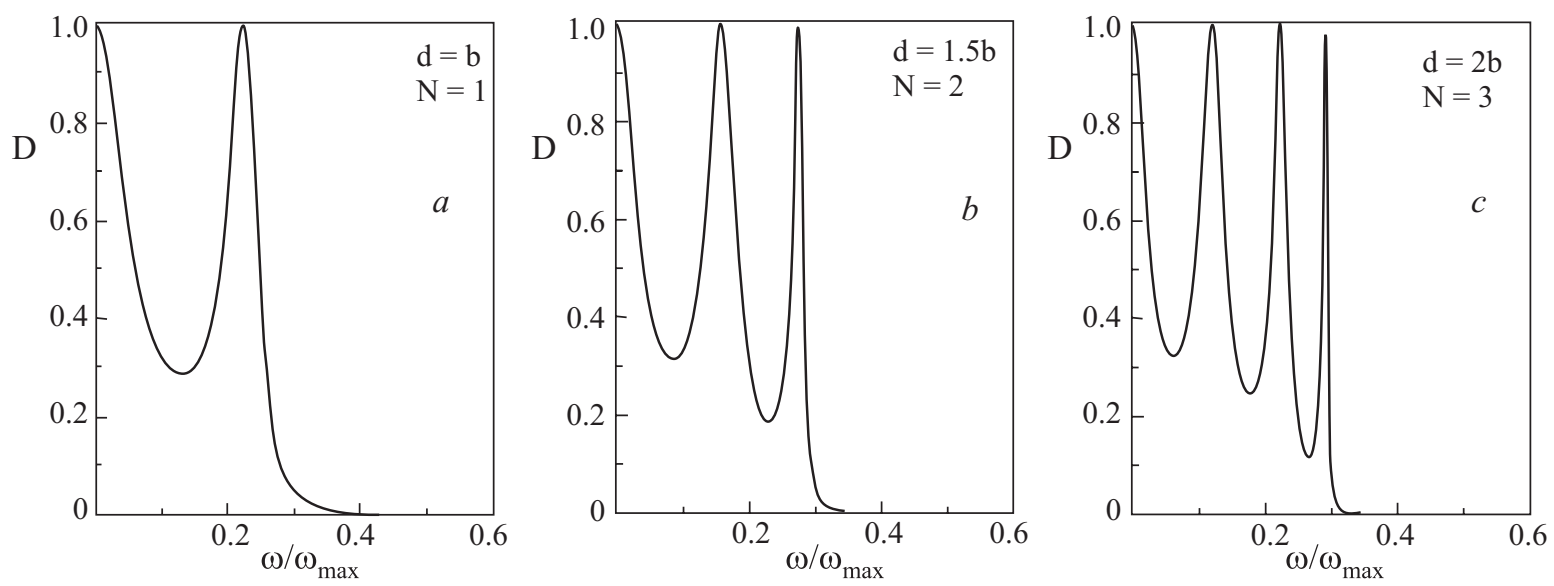

Fig. 4. Frequency dependence of energy transmission coefficients $D$ of $N$ atomic impurity layer: $N=1, b=d(a) ; N=2, b=1.5 d$ (b); $N=3, d=2 b(c)$. 
Using the model of the multichannel resonance transport, we modify the expression (6) in the following way:

$$
\begin{gathered}
\frac{\dot{Q}}{T^{4}-T_{0}^{4}}=\frac{1}{T^{4}-T_{0}^{4}} \times \\
\times \sum_{n=1}^{3} K_{n}\left[\left(\exp \left(3.89 \frac{T_{n}}{T}\left(1+\frac{1}{T_{s}^{2}}\left(T-T_{n}\right)^{2}\right)\right)-1\right)^{-1}-\right. \\
\left.-\left(\exp \left(3.89 \frac{T_{n}}{T_{0}}\right)-1\right)^{-1}\right]+C .
\end{gathered}
$$

The optimal correspondence between the values calculated by this formula and the experimental results was obtained with the following values of parameters:

$$
\begin{gathered}
T_{0}=0.15 \mathrm{~K} ; T_{1}=4.46 \mathrm{~K} ; T_{2}=6.8 \mathrm{~K} ; T_{3}=8.71 \mathrm{~K} ; T_{s}=1.5 \mathrm{~K} ; \\
K_{1}=0.7 \mathrm{nW}, K_{2}=2 \mathrm{nW}, K_{3}=50 \mathrm{nW}, \\
C=49.55 \mathrm{nW} / \mathrm{K}^{4} .
\end{gathered}
$$

The expression (7) takes into account the presence of three channels of the resonance transport. It also accounts for (using an additional term containing the intrinsic temperature $T_{c}$ ) the instability of the intermediate layer of weakly bound impurities near the resonance. The instability of the intermediate layers near the resonance is conditioned by the fact that $u_{0}^{s} / u_{1,2} \gg 1$ (where $u_{0}^{s}$ is displacement of intermediate layer, $u_{1,2}$ are displacements of value layers) [4]. The role of the adsorption-desorption mechanism of impurity ions was discussed in papers $[14,15]$. In Fig. 6 we present experimentally observed temperature dependence of the reduced heat flux in $\mathrm{Si}-\mathrm{Cu}$ point-contacts above $2 \mathrm{~K}$. Results of numerical calculations with the use of expression (6) are presented in Fig. 7. These results indicate that the proposed model describes the experimental results presented in Fig. 6 with a good accuracy. It should be noted that the temperature $T_{S}$ used in calculations corresponds to the binding energy of the impurity layer with contact banks. Temperature $T_{s}$ is by two orders of magnitude lower than the Debye temperature of crystals forming banks of contacts. This is in

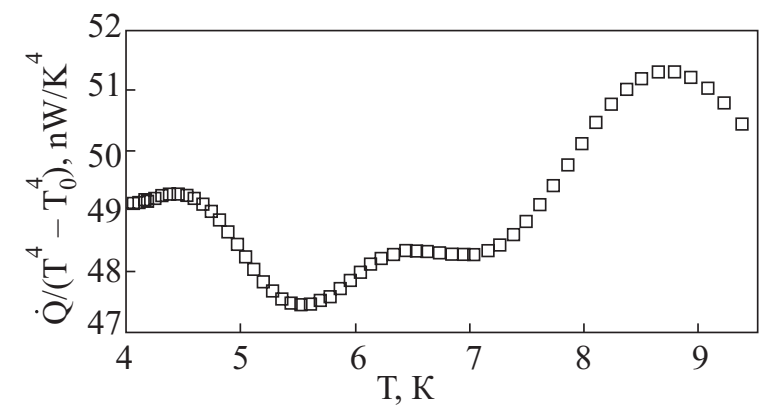

Fig. 6. Experimentally observed temperature dependence of the reduced heat flux in $\mathrm{Si}-\mathrm{Cu}$ point-contacts above $2 \mathrm{~K}[11,12]$.

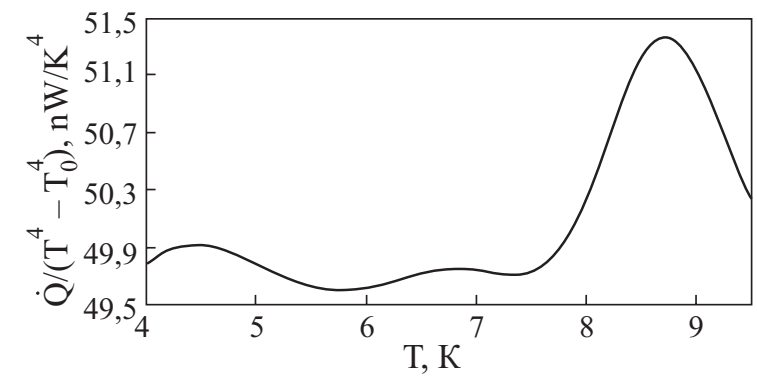

Fig. 7. Results of a numerical calculation within the considered model: $T_{0}=0.15 \mathrm{~K} ; T_{1}=4.46 \mathrm{~K} ; T_{2}=6.8 \mathrm{~K} ; T_{3}=8.71 \mathrm{~K} ; T_{S}=$ $=1.5 \mathrm{~K} ; K_{1}=0.7 \mathrm{nW}, K_{2}=2 \mathrm{nW}, K_{3}=50 \mathrm{nW}, C=49.55 \mathrm{nW} / \mathrm{K}^{4}$.

agreement with the fact that the binding constant of the impurity layer with the banks of contacts is by two orders of magnitude lower than the binding constant in crystals forming this contact [16-18]. Formulae (6) and (7) permit one to evaluate the parameters of different channels of the phonon transport. Coefficients $K_{i}$ are proportional to the areas of different interface layers. If we assume that the channel area with the largest resonance frequency is unity, than we obtain the ratio 1:0.04:0.014 between areas of layers taking part in the resonance transport. When combining formulae (2), (6), (7), we obtain the relation

$$
\left(\frac{T_{\max n}}{T_{\min m}}\right)^{2}=\frac{\rho_{s}^{m}}{\rho_{s}^{n}}=\frac{L_{m}}{L_{n}} .
$$

It follows from the experimental data that

$$
\frac{L_{2}}{L_{3}}=1.8 \pm 0.2, \frac{L_{1}}{L_{3}}=3.8 \pm 0.2,
$$

where $L_{3}$ corresponds to the number of layers with the largest resonance frequency (smallest $\rho_{s}$ ). It follows from the ratio (8) that the lowest frequency channel $(n=1)$ contains the maximum number of layers (about four times more than the high-frequency channel with $n=3$ ), and the channel with the intermediate frequency $(n=2)$ includes two times more layers than the high-frequency channel with $n=3$.

\section{Fano resonance in phonon transport}

The phenomena described in previous section are called «multichannel», but these phenomena are connected with the transmission of phonons through the homogeneous film of finite thickneess $d$ (Fabry-Perot resonance interferometer). Indeed, in the case of the Fabry-Perot interferometer, the number of transmission peaks is determined by the number of the interface intercalant impurity layers (see Fig. 3 for the case of three intercalant impurity layers). For the Fabry-Perot resonances, the transmission peaks have symmetric shape and interaction of incident phonon with the impurity layers results in the resonance transmission enhancement only. 
But the overall picture of the resonance phonon transmittance through the interface intercalant impurity layers becomes much richer if the additional interaction, namely the interaction between the matrix edges across the impurity layer, is taken into account [5]. Such interaction indeed opens new channels for phonon transmission, which in turn results in Fano-like multichannel phonon transmission phenomena [5].

Uve Fano has published in 1961 an article [19] in which he considered phenomena, connected with the interaction of local levels with a continuum spectrum. Below we discuss briefly the manifestation of the Fano resonance for electrons referring to the paper [20]. Authors of Ref. 20 have observed the Fano resonance in a conductance of a quantum wire with a side-coupled quantum dot. In a weak coupling regime, conductance dips due to the Fano resonance have been revealed. In other words, the Fano resonance (or antiresonance) is a consequence of the destructive interference between a quasi-local state and a continuum of states, which correspond, respectively, to the states in the quantum dot and in the quantum wire. It appears that the line shape of the conductance $G$ as a function of electron energy has a form, which is characteristic for the Fano resonance:

$$
G(\varepsilon) \propto(\varepsilon+q) /\left(\varepsilon^{2}+1\right),
$$

where $\varepsilon$ is the energy difference with respect to the position of the resonance level normalized by the resonance line width, and $q$ is the Fano parameter. The Fano parameter represents the degree of distortion, and $q=0$ corresponds to an anti-resonance conductance dip. It was shown in Ref. 20 that when electron energies in the quantum wire and in the quantum dot coincide (the resonance), the destructive interference between the two conductance channels occurs and electron in the quantum wire reflects back and the conductance shows a minimum.

Fano-like resonance can be found for phonons also [5]. One of the authors of the present work has predicted the appearance of the so-called «asymmetric» vibrational mode (with asymmetric displacement pattern) near a composite (laterally-inhomogeneous) planar defect with symmetrical distribution of atoms and binding constants [5]. In the considered asymmetric mode, the vibrational amplitude is (almost) zero in one semi-infinite crystal (half-space) but is nonzero at the interface planar defect and in another half-space. The asymmetric mode can be described as a «linear combination» of symmetrical and antisymmetrical modes with the same polarization, frequency and two-dimensional wave number. To provide the possibility of the asymmetric cancellation of surface traction and of the crossing between two branches in the long-wave and low-frequency domain, one has to take into consideration the direct interaction (bonding) of the ledges of the planar defect (which in general is equivalent to the account for the interaction between non-nearest neighbors in the lattice-dynamical approach). As it was shown in Ref. 5, the additional «phonon transmission channel» through the direct bonds between the ledges of the planar defect, passing over the «locally resonant» impurity atoms, can give rise to the phenomena of the total reflection and anomalous interface absorption (total nontransmission and nonreflection) of the incident long acoustic wave at the «composite» two-dimensional defect. The physical thickness of such two-dimensional defect can be much less than the wavelength of the totally reflected wave. These dynamical phenomena, as well as the existence of the asymmetric vibration mode near a composite planar defect with a symmetric distribution of atoms and binding constants, do not have counterparts in acoustics of layered media, see, e.g., Ref. 21. Later it was experimentally demonstrated that a thin layer of locally resonant material (a monolayer of steel spheres, coated with silicone rubber and embedded in epoxy matrix) indeed can completely reflect an acoustic wave with a wavelength two orders of magnitude larger than the layer thickness [22].

Now we consider the resonance reflection and transmission of phonons through intercalated layer between two semi-infinite crystal lattices. As in Sec. 1, we study a one-dimensional model and consider an infinitely long oscillator chain which contains a substitution impurity atom, weakly linked with the matrix atoms, see Fig. 1. In this system the frequency of quasi-local (resonance) oscillations of the impurity emerges, at which the transmission coefficient through the impurity becomes equal to unity (full phonon transmission through the interface, see Fig. 2). Above this frequency, the transmission of phonons through the interface is strongly suppressed, see Fig. 4. Now we compare these results with the results, which are obtained with an account for the additional force constant $\gamma_{3}$, corresponding to the interaction between non-nearest neighbors passing through the locally resonant impurity layer, see Fig. 1.

In this case it was shown in Refs. 5, 23 that two frequency regions with enhanced phonon transmission are formed, which are separated by a frequency region with reduced phonon transmission if the non-nearest neighbour force constant $\gamma_{3}$ is larger than the weak bounding force constant $\gamma_{2}$, see Fig. 8 .

The picture of phonon transmission through defect atom is completely inverted in comparison with the case of impurity defect formed by weakly linked impurity, shown in Fig. 1. Namely, for $\gamma_{2}<<\gamma_{3} \approx \gamma_{1}$ a strong transmission valley occurs at the same resonance frequency $\omega_{0} \approx \sqrt{2 \gamma_{2} / m_{1}}$, at which there is a transmission maximum for $\gamma_{3}<<\gamma_{2}<<\gamma_{1}$. Moreover, this transmission minimum occurs on the background of almost total phonon transmission through the impurity atom due to the strong inter- 
action of the matrix atoms through the defect layer (with force constant $\left.\gamma_{3} \approx \gamma_{1}\right)$. Such system permits one to make the frequency filter, which reflects the phonons in a very narrow region of frequencies (when heat transmission is minimal at corresponding temperature), while high phonon transmission is observed in other regions of frequencies. It is worth to mention that such inversion of the transmission and reflection spectra in the two limiting cases is directly related to the interference Fano-like origin of the resonance transmission minimum. Similar inversion of the Fano-like transmission and reflection resonances occurs also in the sound transmission through two-dimensional periodic arrays of thin-walled hollow cylinders due to flexural shell vibration modes [24]. It is worth to mention that the nonmonotonous temperature dependence of the reduced heat flux, shown in Fig. 6, which is proportional to the effective phonon transmission coefficient through the $\mathrm{Si}-\mathrm{Cu}$ microcontact, we can also interpret as a result of Fano-like scattering of phonons by some «nanodefects» located at the contact region, cf. Fig. 8.

In paper [25], independently of paper [5], the occurance of Fano-like resonances in scattering of vibrational waves in perturbed quasi-one-dimensional waveguides was predicted. The analogy between electron and phonon scattering was carried out and coefficients of vibrational wave transmission through multichannel quasi-one-dimensional waveguide, made of a stripe of several parallel interconnected oscillator chains, each mass in which is linked to its nearest and next-to-nearest neighbours, was considered in Ref. 25. Coefficients of phonon transmission with asymmetric line shapes were described, but no generalization of the considered effects to phonon propa-
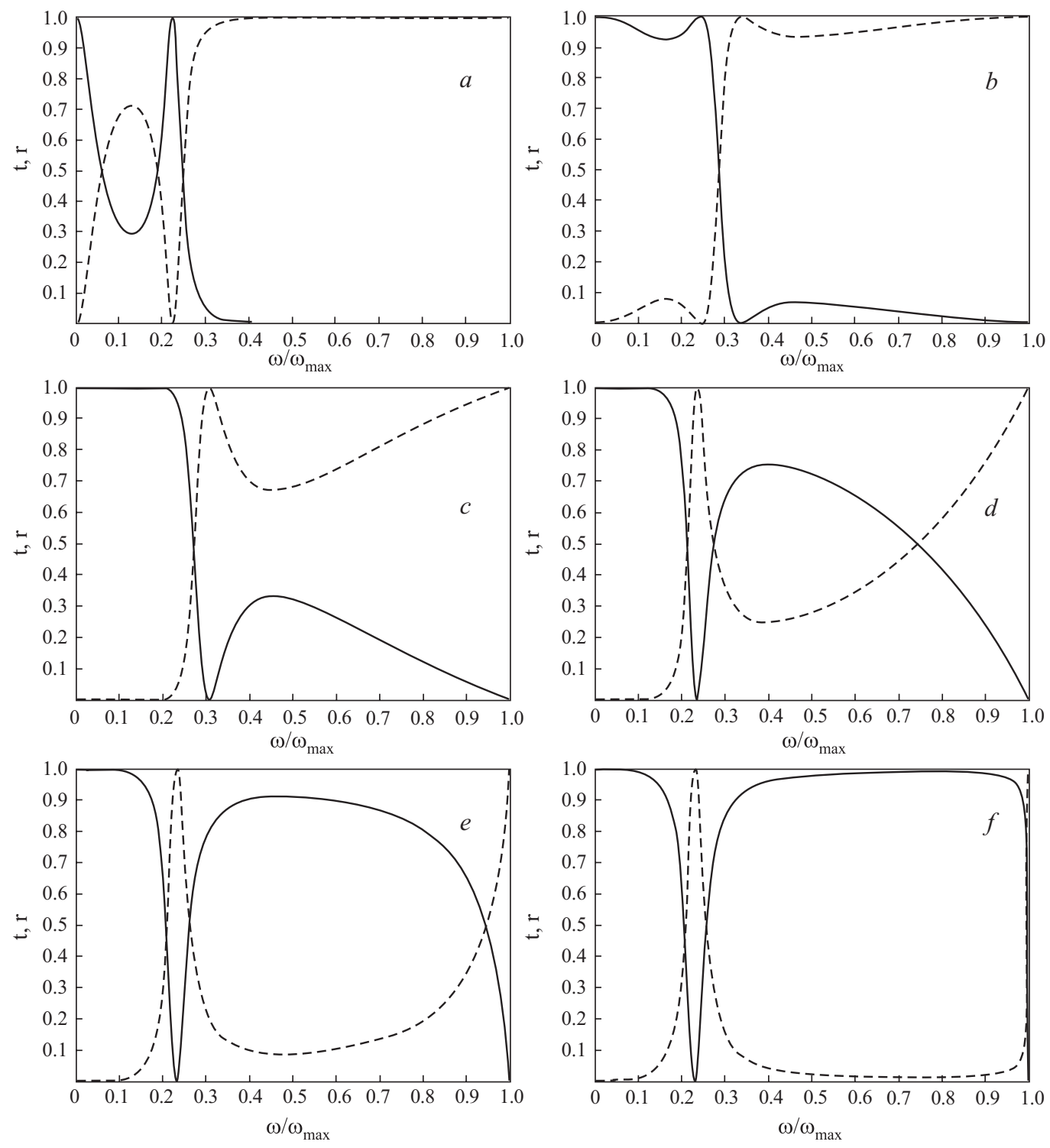

Fig. 8. Coefficients of phonon energy transmission $t$ (solid line) and reflection $r$ (dashed line) through impurity atom: $\gamma_{2}=0.1 \gamma_{1}, \gamma_{3}=0$ $(a) ; \gamma_{2}=0.1 \gamma_{1}, \gamma_{3}=0.15 \gamma_{1}(b) ; \gamma_{2}=0.15 \gamma_{1}, \gamma_{3}=0.3 \gamma_{1}(c) ; \gamma_{2}=0.1 \gamma_{1}, \gamma_{3}=0.5 \gamma_{1}(d) ; \gamma_{2}=0.1 \gamma_{1}, \gamma_{3}=0.7 \gamma_{1}(e) ; \gamma_{2}=0.1 \gamma_{1}, \gamma_{3}=0.9 \gamma_{1}$ $(f)$ versus reduced frequency $\omega / \omega_{\max }$. 
gation in real three-dimensional systems, like considered in Ref. 5 composite (laterally-inhomogeneous) 2D defect in three-dimensional crystal, was proposed in Ref. 25. In paper [26], the problem of phonon reflection from point defect in 1D lattice with nearest-neighbour and next-to-nearest neighbour interactions, including local neighbourhood of the defect, was considered once again. The total phonon reflection from the point defect was described, which was related, similar to the description of this effect in Ref. 5, with the existence of asymmetric vibrational mode in the lattice. The description of total wave reflection from a defect in 1D chain with the interaction between nearest and next-to-nearest neighbour sites was also repeated in recent paper [27].

\section{Magnons and Fano resonance}

As it is well known, there are two channels in the heat transport in magnetic insulators-phonon and magnon channels. Moreover, the theoretical approach for the magnon heat transport is similar to the phonon heat conductivity. So an analogy of the Fano resonance in phonon transport could be considered in the magnon heat transport also. Fano resonance is expected whenever a set of discrete states is mixed with continuum spectrum. Exactly this situation was predicted in the theoretical work [28] for spin-1 magnetic chains with a strong planar anisotropy and an exchange that is either ferromagnetic or antiferromagnetic. Energetic spectrum of these chains consists of three excitation branches approximately in the same energetic domain of magnon dispersion curve, two-magnon continuum and single-ion bound states. The presence of these low-lying magnetic excitations was experimentally observed in EPR experiments [29] and also in thermodynamic properties of $\mathrm{Ni}\left(\mathrm{C}_{2} \mathrm{H}_{8} \mathrm{~N}_{2}\right)_{2} \mathrm{Ni}(\mathrm{CN})_{4}$, usually referred to with the abbreviated symbol NENC [30]. Population of excitations of different branches has a strong magnetic dependence, which opens a possibility to «tune», by external magnetic field, the contribution of different kind of the magnetic excitations to the total magnon heat flux. The interaction between the single-ion bound states and two-magnon continuum could lead to the resonance in the magnon heat conductivity on the background of phonon heat channel. The experimental observation of the Fano resonance in the magnon heat transport is not easy, because the parameters of the magnetic exchange of a studied system must secure the prevalence of the contribution from magnon heat flux over the phonon part of heat flux in the considered temperature and magnetic field range.

It would be interesting to consider similar experiments on spin- 1 chain systems with an additional biquadratic exchange interaction, theoretically already studied in Ref. 31, where the single-ion bound state occurs directly within the continuum, or in spin-1 chains with Ising anisotropy [32].

\section{Effect of anomalous interface absorption}

Our lattice dynamics calculations also confirmed the effect of anomalous interface absorption (almost total nontransmission and total nonreflection) of the acoustic phonon incident on the composite planar defect [5]. To describe this resonance phenomenon, one has to take into account the finite intrinsic dissipative losses of the vibration energy in the vicinity of the planar lattice defect. In the simplest model, this can be accomplished by introducing complex local force constants $\gamma_{2}$ and $\gamma_{3}$, characterizing the defect, whose imaginary parts effectively account for these losses. The effect is more pronounced when a mass $m_{1}^{\text {(edge) }}$ of the outermost atoms at the edges of the matrix, surrounding the impurity layer, is larger than the masses of both the matrix atom, $m_{1}^{\text {(edge) }}>m_{1}$, and the defect atom, $m_{1}^{\text {(edge) }}>m_{2}$. In Fig. 1 , it corresponds to the case when the atoms at the sites $n=-1$ and $n=1$ have a mass $m_{1}^{\text {(edge) }}$, and $m_{1}^{\text {(edge) }}>m_{1}, m_{1}^{\text {(edge) }}>m_{2}$. In Fig. 9 we plot the coefficients of the phonon energy transmission $t$ (dashed line), the reflection $r$ (dotted dashed line) and the interface absorption $A_{s}=1-t-r$ (solid line) at the planar defect with $m_{1}^{\text {(edge) }}=2 m_{1}=2 m_{2}, \gamma_{2} / \gamma_{1}=0.2-i 0.074 \omega / \omega_{\max }$ and $\gamma_{3} / \gamma_{1}=0.4-i 0.963 \omega / \omega_{\max }$, versus reduced frequency $\omega / \omega_{\max }$. The resonance frequency and the conditions for the anomalous interface absorption, with $A_{s} \approx 1$, are the following [5]:

$$
\begin{gathered}
\omega_{0}=\sqrt{\frac{2 \gamma_{2}+\gamma_{2}^{2} / \gamma_{3}}{m_{2}}}=\sqrt{\frac{\gamma_{2}+2 \gamma_{3}}{m_{1}^{(\text {edge })}}}, \quad \frac{m_{1}^{(\text {edge })}}{m_{2}}=\frac{\gamma_{3}}{\gamma_{2}}, \\
\operatorname{Im} \frac{\gamma_{2}+2 \gamma_{3}}{\gamma_{1}}=2 \frac{\omega_{0}}{\omega_{\max }} .
\end{gathered}
$$

The value of $\omega_{0}$, the ratio between masses of edge matrix and defect atoms $m_{1}^{\text {(edge) }} / m_{2}$ and the values of imaginary parts of the local complex force constants $\gamma_{2}$ and $\gamma_{3}$ at the defect, which were used in calculations shown in Fig. 9, exactly fit the analytical prediction given by Eq. (10). The

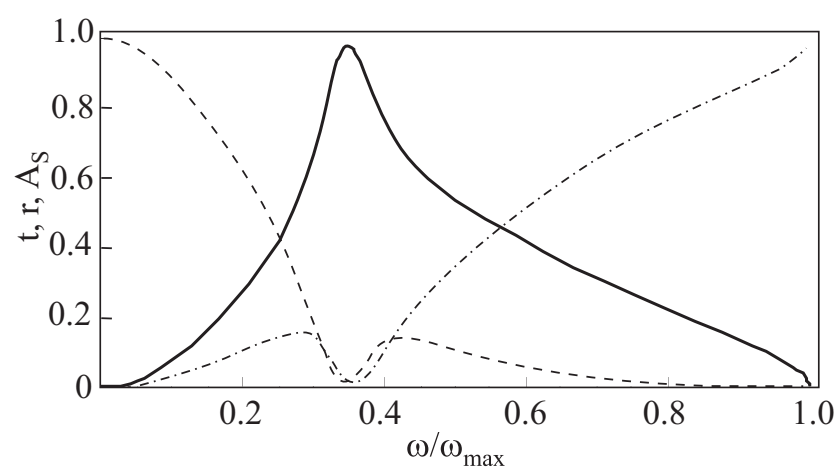

Fig. 9. Coefficients of phonon energy transmission $t$ (dashed line), reflection $r$ (dotted dashed line) and interface absorption $A_{S}=1-t-r$ (solid line) at the planar impurity defect layer with $m_{1}^{(\text {edge })}=2 m_{1}=2 m_{2}, \gamma_{2} / \gamma_{1}=0.2-i 0.074 \omega / \omega_{\max }$ and $\gamma_{3} / \gamma_{1}=$ $=0.4-i 0.963 \omega / \omega_{\max }$ versus reduced frequency $\omega / \omega_{\max }$. 
mass defect of the edge matrix atoms $\left(m_{1}^{\text {(edge) }}=2 m_{1}=2 m_{2}\right)$ sharpens spatial localization of resonance vibrations, which in turn results in the increase of the interface absorption. It is worth mentioning that the phenomenon of anomalous interface absorption at the planar lattice defect is absent for zero non-nearest-neighbour force constant $\gamma_{3}$ (and for $\gamma_{3}<<\gamma_{2}$ ).

\section{Summary}

To summarize, our studies of phonon transport through the interface between two solids in the presence of the intermediate impurity layer have revealed new possibilities for the enhanced heat flux and heat flux with nonmonotonic temperature dependence caused by the resonance and Fano-like interference phonon transmission phenomena.

Moreover, for the materials with the magnon heat transport an additional resonance channel can occur in analogy with the Fano-like interference phenomena for phonons. This kind of magnon heat flux could be observed in some quasi-one-dimensional magnets.

\section{Acknowledgments}

This paper is dedicated to the late Professor Arnold Markovich Kosevich in grateful recollection by one of the authors (A. Feher) of years of gratifying collaboration. Arnold Markovich was the teacher in theoretical physics and excellent mediator of different physical problems for the Kośice Low Temperature Group.

Yu.A.K. acknowledges the support from the Russian Foundation for Basic Research (Grant No. 08-03-00420).

A.F. is indebted to A. Orendáčová and M. Orendáč for helpful discussions and suggestions concerning low dimensional magnets.

E.S.S. has performed part of his work in Kośice at the P.J. Sáfárik University (Slovakia), under the contract No. 20-005204 with the Science and Technology Assistance Agency. Material support of the U.S. Steel DZ Energetika is gratefully acknowledged.

Yu.A.K. and E.S.S. acknowledge also discussions with L.G. Potyomina.

1. I.M. Lifshitz and A.M. Kosevich, Dynamics of Crystal Lattice with Defects, Reports on Progress in Physics, 29, 217 (1966).

2. A.M. Kosevich and V.I. Khohlov, Sov. Phys. Solid State 10, 56 (1968).

3. Yu.A. Kosevich and E.S. Syrkin, Sov. Phys. Solid State 33, 1156 (1991).

4. Yu.A. Kosevich and E.S. Syrkin, Fiz. Nizk. Temp. 20, 660 (1994) [Low Temp. Phys. 20, 517 (1994)].

5. Yu.A. Kosevich, Prog. Surf. Sci. 55, 1 (1997).

6. A.M. Kosevich, E.S. Syrkin, and A.M. Tutov, Fiz. Nizk. Temp. 22, 804 (1996) [Low Temp. Phys. 22, 617 (1996)].
7. Yu.A. Kosevich, E.S. Syrkin, D.A. Semagin, and A.M. Kosevich, J. Eksper. Theor. Phys. 90, 97 (2000).

8. A. Feher, A.A. Mamalui, A.Ya. Dul'fan, E.S. Syrkin, and A.G. Shkorbatov, Fiz. Nizk. Temp. 31, 1211 (2005) [Low Temp. Phys. 31, 921 (2005)].

9. A.M. Kosevich, P.A. Minaev, M.L. Polyakov, and E.S. Syrkin, Fiz. Nizk. Temp. 30, 666 (2004) [Low Temp. Phys. 30, 500 (2004)].

10. A.F. Andreev and Yu.A. Kosevich, Zh. Eksp. Fiz. 81, 1435 (1981) [JETP 54, 761 (1981)] .

11. A.G. Shkorbatov, A. Feher, and P. Stefanyai, Physica B218, 242 (1996).

12. A.G. Shkorbatov, P. Stefanyai, E. Bystrenova, and A. Feher, J. Phys.: Condens. Matter 10, 8313 (1998).

13. A. Feher, P. Stefanyi, P. Zaboi, A.G. Shkorbatov, and T.Z. Sarkisyants, Fiz. Nizk. Temp. 18, 542 (1992) [Low Temp. Phys. 18, 373 (1992)].

14. I.M. Gej'fgat and E.S. Syrkin, Fiz. Nizk. Temp. 4, 672 (1978) [Sov. J. Low Temp. Phys. 4, 324 (1978)].

15. E.T. Swartz and R.O. Pohl, Rev. Mod. Phys. 61, 605 (1989).

16. O.I. Shklyarevskii, I.K. Yanson, and A.A. Lysykh, Fiz. Nizk. Temp. 1, 502 (1975) [Sov. J. Low Temp. Phys. 1, 246 (1975)].

17. L. Koestler, S. Wurdack, W. Dietsche, and H. Kinder, in: Phonon Scattering in Condensed Matter, M. Meissner, and R.O. Pohl (eds.), Springer Berlin, 5, 171 (1986).

18. N.D. Lang, Phys. Rev. Lett. 56, 1164 (1986).

19. U. Fano, Phys. Rev. 124, 1866 (1961).

20. M. Sato, H. Aikawa, K. Kobayashi, S. Katsumoto, and Iye Ya, Phys. Rev. Lett. 95, 066801 (2005).

21. L.M. Brekhovskikh, Waves in Layered Media, Academic Press, New York (1980).

22. Z. Liu, X. Zhang, Y. Mao, Y.Y. Zhu, Z. Yang, C.T. Chan, and P. Sheng, Science 289, 1734 (2000).

23. Yu. Kosevich, L. Potemina, and Ye. Syrkin, in: Book of Abstracts of the 12th International Conference on Phonon Scattering in Condensed Matter "PHONONS 2007», Paris, July 15-20 (2007), p. 161; Yu.A. Kosevich, L.G. Potyomina, and Ye.S. Syrkin, in: Proceedings of the Second International Conference "NONLINEAR DYNAMICS», Dedicated to the 150th Anniversary of A.M. Lyapunov, Kharkov, September 25-28 (2007), p. 138.

24. Yu.A. Kosevich, C. Goffaux, and J. Sanches-Dehesa, Phys. Rev. B74, 012301 (2006).

25. A. Fellay, F. Gagel, K. Maschke, A. Virlouvet, and A. Khater, Phys. Rev. B55, 1707 (1997).

26. A.M. Kosevich and S.E. Savotchenko, Fiz. Nizk. Temp. 25, 737 (1999) [Low Temp. Phys. 25, 550 (1999)].

27. M.I. Tribelsky, S. Flach, A.E. Miroshnichenko, A.V. Gorbach, and Yu.S. Kivshar, Phys. Rev. Lett. 100, 043903 (2008).

28. N. Papanicolaou, A. Orendáčová, and M. Orendáč, Phys. Rev. B56, 8786 (1997).

29. M. Orendáč, S. Zvyagin, A. Orendáčová, M. Sieling, B. Luthi, A. Feher, and M.W. Meisel, Phys. Rev. B60, 4170 (1999).

30. M. Orendáč, A. Orendáčová, J. Černák, A. Feher, P.J.C. Signore, M.W. Meisel, S. Merah, and M. Verdaguer, Phys. Rev. B52, 3435 (1995).

31. T.S. Chiu-Tsao, P.M. Levy, and C. Paulson, Phys. Rev. B12, 1819 (1975).

32. R.P. Hodgson and J.B. Parkinson, J. Phys. C18, 6385 (1985). 